ojs.uv.es/index.php/qfilologia/index

Rebut: 03.05.202I. Acceptat: I5.09.202I

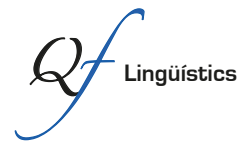

Per a citar aquest article: Carratalá, Adolfo. 202I. "Invertir la vulnerabilidad: el discurso en Twitter de organizaciones neocón y Vox contra las personas LGTBI”. Quaderns de Filologia: Estudis Lingüístics XXVI: 75-94.

doi: $10.7203 / Q F .26 .21979$

\title{
Invertir la vulnerabilidad: el discurso en Twitter de organizaciones neocón y Vox contra las personas LGTBI
}

Reversing vulnerability: the discourse of neocon organizations and Vox on Twitter against LGTBI people

\author{
Adolfo Carratalá \\ Universitat de València \\ adolfo.carratala@uv.es
}

Resumen: Las campañas antigénero, comunes en los últimos años en Europa, cuentan con largo recorrido en España de la mano de organizaciones como Foro Español de la Familia y HazteOir.org, con un discurso contra la igualdad LGTBI compartido por el partido ultraderechista Vox. Esta investigación analiza los tuits publicados por estos tres actores en torno a la población LGTBI durante los dos años siguientes a la entrada de Vox en las instituciones ( $\mathrm{N}=506)$. Los resultados señalan que el discurso dominante responde a una lógica defensiva que trata de legitimar un mensaje discriminador.

Palabras clave: LGTBI; organizaciones neocón; Vox; Twitter; igualdad.

Abstract: Anti-gender campaigns, common in Europe in recent years, have a long history in Spain promoted by organizations such as Foro Español de la Familia and HazteOir.org, with a discourse against LGTBI equality shared by the far-right party Vox. This research analyzes the tweets published by these three actors regarding the LGTBI population during the two years following the entry of Vox into the institutions $(\mathrm{N}=506)$. The results show that the dominant discourse responds to a defensive logic that tries to legitimize a discriminating message.

Keywords: LGBTI; neocon organizations; Vox; Twitter; equality.

\section{Lucha antigénero en España: principales actores}

Europa es la región del mundo en la que los derechos LGTBI se encuentran más desarrollados y emerge, por tanto, como un espacio nítido para los derechos sexuales de la ciudadanía (Fillieule \& Broqua, 2020). Sin embargo, el continente también ha sido escenario en los últimos años del surgimiento 
y despliegue de numerosas campañas antigénero, movilizaciones que han puesto en el punto de mira los derechos de las mujeres y de la población LGTBI (Paternotte \& Kuhar, 20I8). Estas "guerras de género" se corresponden con una "versión generizada" de las llamadas guerras culturales (Alabao, 202I: 398), concepto con el que desde hace años se alude en EE. UU. a los conflictos sociales que colocan la identidad en el centro de la disputa y que se articulan a través de una combinación de política, religión y comunicación, unidas a través de un engarce "emocionalmente muy fuerte" (Lakoff, 2007: I29), y que son el resultado de percibir como amenaza la introducción de programas progresistas y liberales en la estructura social.

Las campañas antigénero, que en muchos países cuentan con el destacado concurso de actores políticos del populismo de derechas, han tenido diferentes recorridos según el contexto, pero coinciden en criticar a la llamada ideología de género y combatir iniciativas como el matrimonio entre personas del mismo sexo y la introducción de la educación afectivo-sexual en los colegios (Paternotte \& Kuhar, 2018). El objetivo de estos actores conservadores, muchos de ellos miembros de la red Agenda Europa (Datta, 20I8; Graff et al., 2019; Ruzza, 202I), ha quedado plasmado en el manifiesto Restaurar el orden natural, que señala necesario "revocar las leyes actuales sobre derechos humanos básicos relacionados con la sexualidad y la reproducción tales como [...] la igualdad de lesbianas, gais, transexuales, bisexuales o intersexuales (LGTBI); o el derecho a cambiar de género o sexo sin miedo a repercusiones legales" (Datta, 20I8: 2).

Aunque el carácter transnacional de estas campañas se observa en países como Alemania, Italia, Polonia, Rusia y Eslovaquia, y el punto de inflexión de $\mathrm{su}$ impacto se identifica con las manifestaciones en contra del matrimonio igualitario que tuvieron lugar en Francia en 2012 (Paternotte \& Kuhar, 20I8), el caso español está considerado como el primer ejemplo europeo de la movilización de los católicos laicos en las calles (Paternotte, 20I5; Paternotte \& Kuhar, 20I8). Su arranque coincide con la aprobación del matrimonio entre personas del mismo sexo en 2005 (Cornejo \& Pichardo, 20I8), momento en el que se inició una amplia ofensiva de los neocones ${ }^{1}$ españoles contra dife-

\footnotetext{
${ }^{\text {I }}$ En el contexto de este estudio, el concepto neocón (de neoconservadurismo) alude al movimiento católico laico español (Díaz-Salazar, 2007) que ha liderado protestas contra legislaciones y reformas progresistas mediante fórmulas movilizadoras propias de las organizaciones que en EE. UU. conforman la Christian Right, protagonistas de numerosas campañas reaccionarias bajo el marco de la guerra cultural (Wilcox \& Larson, 2006). La estrategia de los neocones en España "consiste en la formación de un bloque católico unido dogmáticamente
} 
rentes medidas progresistas del Gobierno de Zapatero, que se prolongaría hasta el fin de la legislatura, en 20II (Alabao, 202I; Carratalá, 20I3).

Aquellas campañas estuvieron protagonizadas por organizaciones de perfil conservador que, animadas por la victoria de los neocones protestantes en la sociedad civil y el Gobierno de Estados Unidos (Díaz-Salazar, 2005: 24), lograron convocar diversas manifestaciones con significativo impacto social y mediático, con el apoyo del Partido Popular y la jerarquía eclesiástica (Cornejo \& Pichardo, 20I7). De entre aquellos actores antigénero, destacan dos (Cornejo \& Pichardo, 20I8). Por un lado, el Foro Español de la Familia (FEF), una red de asociaciones fundada en 2000 que lideró las manifestaciones contra el matrimonio igualitario (Cuesta, 202I). Por otro, la entidad HazteOir.org (HO), surgida en 200I y colaboradora del FEF durante los primeros años, al mismo tiempo que iniciaba su propia movilización contra los derechos sexuales y reproductivos (Cornejo \& Pichardo, 20I8). De perfil de extrema derecha y ultracatólica (Comas d’Argemir, 20I9) y con un posicionamiento claro en contra de las leyes LGTBI (Cuesta, 202I), HO es la organización encargada de la implementación de la estrategia de la Agenda Europa en España (Datta, 20I8).

Los actores antigénero también actúan desde el campo de la política. El partido Vox, fundado en 2013 por exmiembros del PP desilusionados con la estrategia de Rajoy tras alcanzar el Gobierno, es una de las pocas formaciones políticas que pueden ser identificadas por su clara agenda contra los derechos sexuales y reproductivos (Cornejo \& Pichardo, 20I8), la única que en la actualidad cuenta con representación institucional. Vox recoge gran parte del discurso de la ofensiva que supusieron las movilizaciones de la derecha neocón al defender la familia tradicional y heterosexual y negar la existencia de la desigualdad (Castillo Jara et al., 20I9; Ramos, 202I), y ha hecho de la confrontación con las reivindicaciones feministas una de sus banderas (Michavila, 2019), hasta el punto de llegar a poner sobre la mesa la derogación de leyes de género y LGTBI (Ferreira, 2019).

La esfera social y la política no son compartimentos estancos y ambas interactúan de forma manifiesta. Los diferentes actores:

difieren en su composición social, en su naturaleza, en su posición política y en el uso de recursos y estrategias de movilización, pero todos comparten una agenda afín en su pelea contra: el matrimonio y la adopción para parejas del

para crear un polo social público que desde la sociedad civil genere discurso cultural y presión política" (Díaz-Salazar, 2005). 
mismo sexo, [...] la educación sexual y de género, [...] las problemáticas transgénero y transexuales (Cornejo \& Pichardo, 20I8: 530).

El nexo entre HO y Vox es evidente, como demuestra el que la organización ultracatólica premiara a dirigentes de Vox y diera apoyo explícito a la formación en campaña electoral o que varios miembros del partido también figuren como socios de esta entidad (Alabao, 202I; Comas d'Argemir, 2019; Cuesta, 202I). En palabras de Tamayo (202I), "la actual alianza entre las organizaciones políticas y sociales de la extrema derecha, apoyadas por el ultraliberalismo, y los movimientos cristianos integristas" es "la nueva religión": el "cristoneofascismo".

\section{La estrategia retórica de la autovictimización}

Uno de los principales instrumentos políticos empleado por estos actores es el concepto ideología de género, que definen como el matriz ideológico de diferentes reformas a las que se oponen, relacionadas con debates ciudadanos en torno a la intimidad y la sexualidad, incluyendo derechos LGTBI y la educación afectivo-sexual (Paternotte \& Kuhar, 20I8). Este concepto, que se ha mostrado como un potente movilizador social y uno de los elementos discursivos más exitosos para unir luchas antigénero (Alabao, 202I), ha impregnado el mensaje impulsor de estas campañas en diferentes contextos. En Estados Unidos, la ideología de género se convirtió en la argucia que inventó la Mayoría Moral para luchar contra los derechos de las mujeres y personas LGTBI tras la Conferencia Internacional sobre Población y Desarrollo celebrada en El Cairo en I994 y la Conferencia Mundial sobre la Mujer que acogió Pekín en I995 (Fillieule \& Broqua, 2020). En España, la expresión apareció por primera vez en una instrucción pastoral de la Iglesia católica en 200I titulada "La familia, santuario de la vida y esperanza de la sociedad" (Cornejo \& Pichardo, 20I8). En el contexto latinoamericano, este "peligroso" concepto también ha sido incorporado en la agenda de los grupos políticos más conservadores, que atacan frontalmente las iniciativas y reformas en favor de las mujeres y la población LGTBI, ya que son vistas como "una imposición de un sistema de creencias que amenaza los valores cristianos y corrompe la sociedad" (Comas d'Argemir, 20I9: 48).

Uno de los elementos básicos de la estrategia de los protagonistas de las campañas antigénero descritos es, en efecto, la difamación de los oponentes y de las instituciones que los apoyan (atribuyendo, por ejemplo, al llamado lo- 
bby gay acciones intimidatorias y violencia física), la presentación de sus ideas en términos de derechos (imitando de forma artificial el lenguaje característico de los derechos humanos) y la desacreditación de la condición de víctimas de sus oponentes para posicionarse ellos mismos como las nuevas víctimas reales de la "revolución cultural" (señalando, por ejemplo, una supuesta discriminación e intolerancia contra los cristianos) (Datta, 20I8). Esta dinámica se corresponde, según Fillieule \& Broqua (2020), con el efecto isomorfo: intentar imitar el enmarcado sobre asuntos sociales de sus oponentes cuando se comprueba que resulta eficaz.

La estrategia de autovictimización es elemental en el discurso de los actores antigénero. Una de las claves de su manera de hacer frente a las reformas políticas a las que se oponen es presentarse a sí mismos como los verdaderos defensores de la gente oprimida, de una mayoría que se encuentra silenciada por élites y lobbies poderosos, así como los salvadores de una autenticidad nacional frente a poderes internacionales (Paternotte \& Kuhar, 20I8). La fórmula busca conectar con la idea del derecho agraviado señalada por Kimmel para explicar el enojo de muchos hombres estadounidenses en los últimos años, que se ven víctimas de la igualdad racial y de género, que viven como una erosión de sus legítimos privilegios al sentir que "aquellas ventajas sobre las que creíamos tener derecho nos han sido arrebatadas por fuerzas invisibles mucho más grandes y poderosas" (2019: 49). De hecho, Vox ha conectado con un electorado, "mayoritariamente masculino, que se siente agraviado por unas políticas fruto de lo que califican de «ideología de género»” (Michavila, 2019: 32).

Esta lógica de victimización que permite crear la figura de la víctima que se rebela y se torna resistente es esencial en el mensaje de Vox frente a la llamada dictadura progre (Juste, 202I) y constituye una noción central de su discurso antifeminista (Rivas Venegas, 202I), que llama a la urgente protección de los hombres y que acusa a los colectivos feministas la responsabilidad de una división social entre hombres y mujeres (Luque Ortiz \& Cano Alarcón, 202I). También se observa, asimismo, en el lenguaje defensivo que emplean organizaciones como el FEF (Cornejo \& Pichardo, 20I8). Este discurso permite sortear un mensaje explícitamente homófobo, como hizo Printemps Français en su campaña contra el matrimonio igualitario en 2013, cuando apuntó a un nuevo totalitarismo promovido por las élites (de izquierdas y liberales) que buscaba destruir la familia heterosexual y la civilización como la conocemos (Mayer et al., 2016). 
La voluntad de armar un discurso defensivo supone sumar la idea del pánico moral, representado por una condición, episodio, persona o grupo de personas que emerge en la esfera pública para ser definido como clara amenaza a los valores e intereses sociales (Cohen, 2004) y que a menudo incorpora la imagen de los menores como sus principales víctimas y, por tanto, sujetos merecedores de protección:

The protectionist tendency is clearly mobilized by moral panics. The grounds are that children are increasingly, to use a social work term, «at risk». [...] All moral panics about childhood identify a risk from adults; define children as vulnerable to them; proclaim the need to defend innocence of childhood against corruption; and require authority to intervene to protect children (Critcher, 2003: 158).

La ideología de género se convierte así en "un buen activador de los «pánicos morales» del conservadurismo cultural” (Alabao, 202I: 40I) mediante el uso recurrente de la imagen de niños inocentes y en peligro con el objetivo de legitimar sus demandas, establecer la validez de los asuntos que denuncian, suscitar preocupación entre la población general y atraer la atención de los medios (Paternotte \& Kuhar, 20I8). Como indica Comas d'Argemir (20I9: 53): "l'estratègia és produir confusió i pànic moral, ja que es propugna que la ideologia de gènere destrueix la familia $i, a m b$ això, amenaça la humanitat".

\section{LGTBIfobia en red: incremento del odio y respuesta legislativa}

El discurso de los defensores de campañas antigénero ha encontrado en la red un espacio de difusión privilegiado. Los promotores de esta operación son extremadamente activos en la web y se benefician de las posibilidades que ofrecen las tecnologías de la información y la comunicación (Paternotte \& Kuhar, 20I8). En concreto, las redes sociales acompañan este juego retórico al convertirse en reproductoras del discurso que viene desde el terreno de lo político (Rivas Venegas, 202I), hasta llegar a constituir un caldo de cultivo para la proliferación de noticias falsas y mensajes de odio (Juste, 202I). Además, la lógica androcéntrica del Internet más reciente, dominado por redes sociales como Twitter, refuerza "la resistencia simbólica de ciertos sectores sociales ante el avance del feminismo" (Villar-Aguilés \& Pecourt García, 202I: 34), mediante campañas de acoso y desprestigio en manos de actores antigénero como HO o Vox. 
El primero ha destacado desde su nacimiento por desarrollar buena parte de sus acciones en el medio digital, que a menudo funciona como un complemento perfecto de sus campañas en el espacio físico, como ocurrió con la acción "Los niños tienen pene. Las niñas tienen vulva", desplegada en 2017 (Simancas González \& Marín Dueñas, 20I9). Vox, por su parte, ha destacado como "la formación que mejor ha sabido usar las redes para crecer y llegar a la ciudadanía" (Luque Ortiz \& Cano Alarcón, 2021: 100) y entender que el uso que hace de las redes sociales y su constante agitación en estas, sobre todo a través de Twitter, es fundamental para comprender su éxito (Ramos, 202I). También el FEF ha dedicado buena parte de sus recursos humanos y económicos a garantizar su presencia en redes sociales (Cornejo \& Pichardo, 2018). La acción colectiva de este tipo de organizaciones neocón encuentra en Twitter un nuevo foro en el que articular sus protestas, por ejemplo, de carácter educativo (Carratalá, 2020).

Es precisamente a través de internet y las redes sociales como se distribuye principalmente el discurso de odio en España. Según los datos que registra desde 2015 el Ministerio del Interior, los hechos relacionados con la difusión de este discurso han crecido en los últimos años, desde los II7 casos reflejados en su primer informe hasta los 189 episodios recogidos en el último anuario hecho público, referente a 2020. El i6,9\% de ellos se encuentran vinculados con la discriminación por orientación sexual o identidad de género. Este último informe señala, asimismo, que la mayor parte de los discursos de odio de los que se tiene constancia oficial se canaliza a través de internet $(45 \%)$ y las redes sociales $(22,8 \%)$ (Ministerio del Interior, 202I).

En paralelo al incremento de los delitos y los discursos de odio en España, diversas comunidades autónomas han puesto en marcha leyes orientadas a proteger los derechos y a garantizar la igualdad de las personas LGTBI que viven en sus territorios, así como a luchar contra su discriminación. La suma de estas normas, que contemplan tanto leyes dirigidas a la población LGTBI en general como leyes orientadas a las personas trans en particular, eleva a I9 las iniciativas de este tipo aprobadas entre 20II y 2020. Estas leyes otorgan una atención especial a la contención del discurso discriminatorio por razón de orientación sexual o identidad de género; sin embargo, el foco se sitúa principalmente sobre los medios de comunicación social, a los que consideran actores sensibles y claves para alcanzar la igualdad de trato y no discriminación, y apenas se contempla cómo actuar ante su canalización en redes sociales (Carratalá \& Herrero-Jiménez, 20I9). 


\section{Metodología}

Este trabajo tiene como objetivo caracterizar el discurso de los principales actores antigénero en España en torno a la igualdad de las personas LGTBI en un contexto de institucionalización de la ultraderecha y de crecimiento del discurso del odio. Dada la simbiosis en términos de agenda y comunicación entre la esfera social y política neoconservadoras, y la importancia que las redes sociales tienen para la canalización de su mensaje, la investigación sitúa como objeto de estudio la producción discursiva en Twitter de las organizaciones Foro Español de la Familia y HazteOir.org y del partido político Vox durante los dos años siguientes a que esta formación accediera, por primera vez, a una cámara legislativa en España. Así, el período de análisis comprende desde el 2 de diciembre de 20I8, fecha de las elecciones autonómicas de Andalucía en las que Vox obtuvo I2 escaños, al I de diciembre de 2020 , momento en el que la formación ya contaba con representación en I2 parlamentos regionales, así como también en el Senado, el Congreso de los Diputados y el Parlamento Europeo.

El estudio busca dar respuesta a las siguientes preguntas de investigación:

PII: ¿Qué presencia tuvieron los temas relacionados con la igualdad de las personas LGTBI en la comunicación de estos actores y cuáles fueron los asuntos que dominaron en su discurso?

PI2: ¿Responde el mensaje de estas organizaciones a una lógica victimista? En ese caso, ¿sobre qué elemento trata de construirse su discurso defensivo?

$\mathrm{PI}_{3}$ : ¿Se observan interpelaciones entre los diferentes actores analizados a través de su discurso en Twitter?

Para poder responder a estas preguntas, se ha llevado a cabo una búsqueda avanzada limitada al periodo de investigación en las tres cuentas que los actores sometidos a estudio tienen en Twitter (@ForoFamilia, @hazteoir, @ vox_es) por medio de diferentes palabras clave ${ }^{2}$ para obtener el conjunto de tuits que conforman el corpus de análisis. Tras revisar los contenidos de las publicaciones recuperadas (768), se descartaron aquellas que no se correspondían con mensajes vinculados a la realidad LGTBI, al quedar circunscritos a otros temas como la violencia de género o la lucha feminista, lo que supuso limitar el corpus definitivo a 506 tuits (tabla I), la mayor parte de ellos (75\%)

\footnotetext{
${ }^{2}$ La ecuación de búsqueda contempló las siguientes palabras: LGTBI OR género OR homosexualidad OR homosexual OR gay OR lesbiana OR bisexualidad OR bisexual OR transexualidad OR transexual OR trans OR intersexualidad OR intersexual.
} 
producidos por la organización HazteOir.org, que han sido sometidos a un análisis de contenido cuantitativo, a través de estadística descriptiva, y cualitativo, con el propósito de conocer la transitividad y la estructura lógica que encierra el discurso de estos actores.

Someter el corpus de tuits a un análisis de contenido de tipo cuantitativo (Bardin, I986) facilita observar la presencia y frecuencia de determinadas características y elementos con el objetivo de conocer qué rasgos resultaron dominantes en el mensaje de estos actores. Así, se han considerado las siguientes variables categóricas, algunas de ellas definidas a partir de una primera exploración parcial del corpus, para poder obtener datos con los que dar respuesta a las tres preguntas de investigación planteadas: año, mes, tema dominante (ley LGTBI, ley trans, educación, transexualidad, menores, ideología de género, otros), victimización (sí, no), agravio denunciado (adoctrinamiento, corrupción, discriminación, engaño, recorte de derechos, totalitarismo, otros) y mención a partidos políticos (PSOE, PP, UP, Vox, Cs, otros).

El examen cualitativo del corpus responde a una aproximación propia de los estudios críticos del discurso. Como indica Van Dijk, esta perspectiva de análisis permite estudiar "la forma en la que el abuso de poder y la desigualdad social se representan, reproducen, legitiman y resisten en el texto y el habla en contextos sociales y políticos" (2016: 204) mediante su enfoque en problemas sociales y cuestiones políticas, como en este caso supone la discriminación de las personas LGTBI. La adopción de esta técnica de análisis tiene como objetivo dar respuesta a la PI2 y, en este sentido, el examen cualitativo de los tuits atenderá fundamentalmente a dos características de los textos, vinculadas al ámbito de la sintaxis y del léxico. En primer lugar, el elemento lingüístico de la transitividad facilita reconocer el modo en que el discurso de los actores analizados representa el mundo a partir de la estructura de los tuits: qué procesos describe, qué participantes incorpora y qué circunstancias vincula a esas representaciones (Fairclough, I995). En particular, con el objetivo de identificar una posible lógica victimista en los mensajes, resultará especialmente útil conocer qué rol (agentes/pacientes) ocupan los actores que en ellos aparecen (Fowler, I99I) y así considerar qué tipo de relación queda establecida entre los enunciadores y la comunidad LGTBI. En segundo lugar, los términos empleados por los actores analizados podrán revelar presuposiciones e implicaciones que permitirán ir más allá del significado explícito, evidente, de los tuits (Richardson, 2007). 


\begin{tabular}{|l|r|r|r|r|}
\hline \multicolumn{1}{|c|}{ Palabra clave } & \multicolumn{3}{c|}{ Organización } & Total \\
\hline & FEF & HO & Vox & \\
\hline LGTBI & 89 & 191 & 4 & 284 \\
\hline Género & 20 & 134 & 7 & 161 \\
\hline Homosexualidad & 1 & 5 & & 6 \\
\hline Homosexual & & 17 & & 17 \\
\hline Gay & 3 & 17 & 1 & 21 \\
\hline Lesbiana & 1 & & & 1 \\
\hline Transexualidad & & 3 & & 3 \\
\hline Transexual & & 5 & & 5 \\
\hline Trans & & 8 & & 506 \\
\hline Total & 114 & 380 & 12 & 8 \\
\hline
\end{tabular}

Tabla I. Número de tuits por organización y palabra clave

\section{Resultados}

\subsection{La cuestión LGTBI en el discurso neocón en Twitter}

El análisis de la evolución de la difusión de mensajes vinculados con las personas LGTBI a lo largo del periodo de estudio (figura I) evidencia una clara irregularidad del discurso desde el punto de vista diacrónico dado que su aparición se concentra en determinados momentos mientras que en otros apenas tiene incidencia. En primer lugar, y más allá de las diferencias entre los tres actores analizados que recoge ya la tabla con el detalle de la muestra, puede observarse un claro desequilibrio entre los años 20I9 y 2020 , dado que en este último se redujo de manera muy notable el número de publicaciones de los tres actores analizados en relación con el tema de estudio. El $78 \%$ de los tuits examinados se publicaron en 2019 .

Mientras que los datos relacionados con la difusión de mensajes de Vox no permite identificar episodios de especial concentración del discurso, la frecuencia de publicación de FEF y HO sí posibilita la delimitación de determinados picos de publicación en ciertos meses, que quedan atravesados por informaciones de actualidad o acciones de estas entidades que explican la elevada incidencia de tuits vinculados a la población LGTBI. En el caso de FEF, la mayor parte de sus tuits se da en los meses de: febrero de 2019 (coincidiendo con varias noticias relacionadas con la tramitación de un proyecto 
de igualdad LGTBI en el Congreso, el adelanto de las Elecciones Generales y discursos de diferentes actores políticos, como la líder de Vox, Rocío Monasterio, sobre los talleres LGTB en los colegios, a los que reacciona este grupo); junio de 20I9 (junto a críticas a la celebración del Orgullo LGTBI, se comentan informaciones varias sobre la hija trans de Nacho Vidal o el asesinato de un menor a manos de sus madres, dos mujeres lesbianas); octubre de 2019 (fundamentalmente marcado por la apertura de la consulta pública sobre la Ley LGTBI de Castilla y León) y enero de 2020 (otra vez, a propósito de un nuevo anteproyecto de ley presentado en Castilla y León junto con noticias sobre la formación del nuevo Ejecutivo central y en torno al debate del PIN parental en Murcia $\left.{ }^{3}\right)$.

En el caso de HO, los picos de incidencia son algunos más, pero destacan especialmente los tuits publicados en los seis siguientes meses: febrero de 2019 (a partir, sobre todo, de la noticia sobre un policía de Logroño supuestamente acosado por "grupos LGTBI"); junio de 2019 (relacionados con la Ley LGTBI de Madrid y el despliegue de la bandera LGTBI por el Ayuntamiento de la capital); julio de 2019 (junto con el Orgullo y la polémica de la bandera, se abre paso una campaña contra el supuesto adoctrinamiento LGTBI de Disney); septiembre de 2019 (muy destacado, con ocasión de la puesta en marcha de la campaña sobre el PIN parental \#MisHijosMiDecisión); diciembre de 20 I9 (ligados, en su mayoría, con una petición para frenar la Ley LGTBI de Cantabria y otra para la retirada de la película "La primera tentación de Cristo" de Netflix, así como diversos proyectos de presupuestos públicos) y junio de 2020 (ley trans).

\footnotetext{
3 El comúnmente llamado PIN parental (expresión empleada por las organizaciones objeto de estudio en los tuits que componen el corpus) es una de las iniciativas políticas con las que el partido Vox ha tratado de negociar su apoyo parlamentario a gobiernos presididos por representantes del PP. En el acuerdo para la aprobación de los presupuestos de 2020 para la Región de Murcia firmado entre PP, Ciudadanos y Vox, aparecía reflejado como "el establecimiento de una autorización expresa de las familias para la participación de sus hijos en actividades complementarias". Voces contrarias a esta medida la consideran un veto educativo a la asistencia de menores a talleres y charlas sobre contenidos como la violencia de género o la diversidad afectivo-sexual.
} 


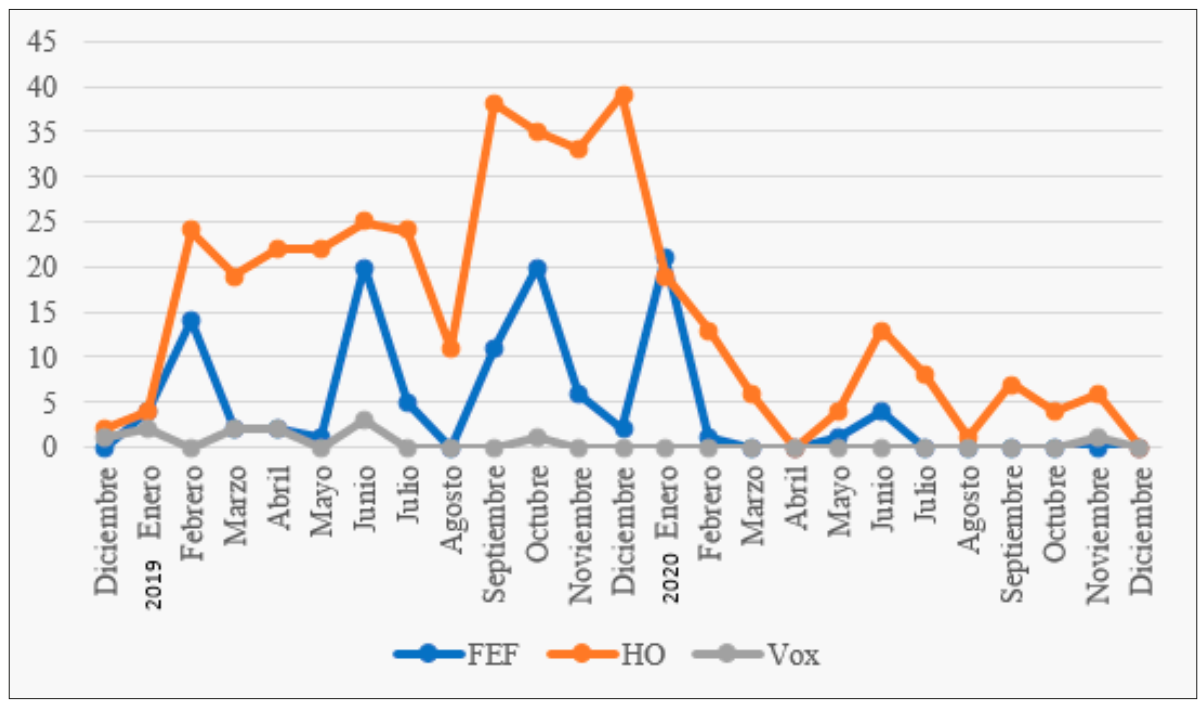

Figura I. Evolución de tuits relacionados con la igualdad de las personas LGTBI en los perfiles estudiados

Si se observa el conjunto de tuits que conforman el corpus de análisis, los temas más frecuentes son aquellos que guardan relación con la tramitación de leyes relacionadas con la igualdad de las personas LGTBI (40,5\%), tanto a nivel autonómico como estatal, y los vinculados a cuestiones educativas $(26 \%)$. El resto de asuntos abordados son de diferente índole e incluyen, a menudo, comentarios a publicaciones sobre noticias de actualidad o campañas de movilización orientadas a objetivos específicos, como la retirada de una bandera LGTBI o la crítica a la financiación con presupuesto público de organizaciones que trabajan por la igualdad de estas personas. Analizado cada actor de manera independiente, se observan ligeros matices en el peso que otorgan a cada tema. Así, Vox (58\%) y, sobre todo, FEF (73\%) ponen especialmente el foco en la tramitación legislativa de derechos LGTBI, mientras que el asunto dominante en el perfil de $\mathrm{HO}$ es el relacionado con la educación (3I,8\%).

\subsection{La construcción de la autovictimización}

El análisis de la transitividad, explícita o a menudo presupuesta en los tuits examinados, permite concluir que prácticamente 3 de cada 4 mensajes responden a una lógica victimista (74,3\%). Los actores examinados hablan desde 
la posición de una mayoría oprimida, que ocupa el rol paciente en el discurso, frente a un grupo minoritario que ejerce presión y discriminación. Aunque algunas veces las personas LGTBI son claramente señaladas como causantes de ese daño ("Grupos LGTBI y el concejal socialista de Logroño Kilian Cruz acosan a este policía de Logroño por proteger a tus hijos", HO, I2/O2/20I9), la mayor parte de las ocasiones la construcción del discurso evita situar directamente a personas LGTBI como sujetos agentes de ese agravio. En su lugar, se opta por fórmulas que asignan a seres inanimados (campañas, celebraciones, leyes...) esa capacidad de ejercer perjuicio, aunque con evidente vinculación a esta minoría social, como ilustran estos dos ejemplos: "!!250.000 firmas apoyan esta petición contra el Orgullo Gay en DisneyLand. Nuestra portavoz, Inmaculada Fdez., pide a @WaltDisneyCo que ‘defiendan la inocencia de los niños, que no hay que hipersexualizarles ni adoctrinarles en prácticas sexuales de ningún tipo" (HO, 3I/05/20I9) y “Si la 'ley LGTBI' se frena en Castilla y León es porque los ciudadanos saben que es un ataque a los niños y a las familias y están presionando a los políticos" (HO, I5/II/20I9).

Un tuit publicado por FEF el I7 de octubre de 20I9, en el marco de su campaña en contra de la normativa discutida en Castilla y León, es muy elocuente al respecto: "Las leyes LGTBI aprobadas en el mundo sólo traen: discriminación, imposición ideológica, privilegios a minorías, inseguridad jurídica, recorte de derechos y libertades fundamentales". La lógica discursiva de plantear el mensaje desde la sumisión es general en el conjunto de los actores sociales, aunque con peso desigual (se identifica en el $67,5 \%$ de los tuits de FEF y en el 77,6\% de los publicados por $\mathrm{HO}$ ), y resulta algo menos presente, aunque también es significativa, en el perfil de Vox (4I,7\%).

Una categorización de los mensajes que comparten una retórica de autovictimización permite considerar las cinco causas principales de los agravios que los actores analizados asegurarían sufrir por el avance en la protección legal y la igualdad social de las personas LGTBI: adoctrinamiento $(35 \%)$, recorte de derechos $(24 \%)$, discriminación (I0,3\%), totalitarismo (8\%) y corrupción de menores $(5,6 \%)$. Otros motivos, como la anticonstitucionalidad de las normas, su inseguridad jurídica o el perjuicio cultural tienen un peso menor.

La clasificación de los tuits de cada actor analizado de acuerdo con estas categorías muestra ligeras variaciones: la mayor parte de los mensajes de FEF $(38 \%)$ y Vox $(60 \%)$ en los que se advierte un discurso victimista apuntan a la discriminación que sufrirían, en su opinión, las personas no LGTBI en el actual contexto social. La idea de que toda ley dirigida a proteger a este grupo social resulta discriminatoria es recurrente en los tuits publicados por FEF. 
Los mensajes "En Asturias preparan una nueva ley que confunde igualdad con discriminar a la mayoría. Una ley LGTBI que, en realidad, penalizará a las personas de este colectivo que no comparta sus postulados" (I7/OI/20I9), “@Junta_CyL inicia consulta popular para tramitar la ley LGTBI regional. Una norma innecesaria, discriminatoria y fuente de inseguridad jurídica" (25/09/2019) y "900 personas ya han votado a favor nuestras alegaciones a la Ley LGTBI que.@Junta_CyL quiere tramitar a la carrera y presentó con nocturnidad y alevosía. Una norma innecesaria que acaba con la igualdad de los ciudadanos según su orientación sexual" (I8/0I/2020), son algunos ejemplos. El partido político Vox atribuye un carácter excluyente a la celebración del Orgullo LGTBI: “@Santi_ABASCAL sobre el veto de los lobbies LGTBI a PP y Ciudadanos en el desfile del Orgullo Gay: «El PP y Ciudadanos entienden ahora las exclusiones que viene sufriendo VOX»" (I2/06/2019).

$\mathrm{HO}$, por su parte, pone el acento en el carácter adoctrinador ( $42 \%)$ y el recorte de derechos (23\%) que implicarían las acciones en favor de diversidad afectivo-sexual y de identidad de género en la sociedad española. La primera de estas estrategias aparece ligada de manera estrecha al discurso en torno a la educación ("iNo permitas que los activistas \#lgtbi entren en las escuelas para adoctrinar a los niños en ideología de género!", II/Io/20I9) mientras que la supresión de derechos se vincula a la tramitación de normativas para proteger a las personas LGTBI, que contemplan como una amenaza a la libertad de expresión y califican como leyes "mordaza", tal y como ilustran estos tuits: “.@PabloCasado tiene la oportunidad durante la convención del @ PPopular de recolocar al partido y posicionarlo contra las leyes de violencia de género discriminatorias, las leyes mordaza LGTBI, las leyes de memoria histórica y la actual ley del aborto" (o9/or/20I9), "Detén la ley mordaza LGTBI de Cantabria” (24/12/20I9), "Podemos impulsa de nuevo la Ley 'mordaza' LGTBI" (25/06/2020).

La autorrepresentación como víctimas frente a un otro violento se observa con claridad en el modo en que su discurso subvierte la lógica estructural del sistema cisheteropatriarcal para situar a las personas LGTBI como representación de una amenaza a la igualdad. Estas, incorporadas a menudo en el mensaje bajo la fórmula de "Lobby LGTBI" o "Grupos LGTBI" (presente en torno al $8 \%$ de los tuits de FEF y HO), dejan de ser víctimas de acoso para pasar a ser acosadores. HO asigna este carácter al "lobby \#LGTBI" el I3/O2/20I9 ("Más de II.000 personas apoyan a este policía acosado por Grupos LGTBI y el concejal socialista de Logroño Killian Cruz por proteger a tus hijos") y el 02/04/2019 ("Todo nuestro apoyo a monseñor Reig Pla, obispo de @diocesi- 
salcala. Un \#obispo valiente que lleva décadas ayudando a las \#personas a pesar del acoso del lobby \#LGTBI"), un día antes de hablar de "cacería \#LGTBI": “.@EspejoPublico entrevistará este martes a las ıo,I5 a @tgnoblejas, portavoz de @hazteoir, para hablar de la cacería \#LGTBI y de la @ComunidadMadrid contra el \#obispo de la @diocesisalcala Juan Antonio Reig". Esta organización también responsabiliza a esta minoría de llevar a cabo agresiones ("El lobby \#LGTBI vuelve a atacar a Elena Lorenzo", HO, 08/02/2020) y comportarse como "auténticos matones" ("Los lobbies LGTBI se comportan como auténticos matones”, HO, I6/10/2020).

La dicotomía que estructura este discurso exige completarlo con la figura del sujeto que sufre la amenaza LGTBI, su víctima, con la que aparentan identificarse estos actores y dicen defender. En ocasiones, aparece de manera explícita, como en un tuit publicado por $\mathrm{HO}$ el 27/OI/2020: "Este miércoles presentaremos un servicio de atención a víctimas de ideología de \#género en las aulas". Su representación también se corresponde a menudo con la figura del menor, la mayor expresión posible de vulnerabilidad, lo que favorece la construcción del pánico moral. Los mensajes de HO dan voz, en esta línea, a una asociación llamada Los Niños Son Intocables que pide "proteger" a los menores "frente al \#adoctrinamiento sexual que impone la ideología de género" (I2/04/2019) y reclaman "dejad a los niños, en paz" en otro tuit a propósito de la celebración del Orgullo Gay en el parque Disneyland (31/05/2019). El discurso de esta organización, próximo a la denuncia de la corrupción de menores, se recrudece con tuits que llegan a señalar que "el adoctrinamiento en ideología de género es abuso infantil” (I8/II/20I9) y que aluden incluso a la pedofilia, alimentando así un falso vínculo tradicionalmente empleado para estigmatizar y condenar la homosexualidad. Al mismo tiempo, incrementan el tono alarmista de su mensaje: "Un grupo de pedófilos quiere desfilar en el orgullo gay de Ámsterdam bajo la bandera LGTBI en un intento de normalizar los abusos sexuales a menores" (31/07/2019).

\subsection{Interacción político-social en la comunicación en Twitter}

El análisis de los tuits objeto de estudio muestra ejemplos de interpelación de los actores antigénero de perfil social (FEF, HO) al actor antigénero político (Vox), pero no en dirección contraria. El partido de ultraderecha no incorpora a su discurso a las organizaciones que comparten mensaje con él en torno a la realidad LGTBI. Estas entidades, sin embargo, sí aluden a diversos partidos 
políticos del arco parlamentario español, entre los que se encuentra Vox. El $20 \%$ de los tuits publicados por la organización FEF relacionados con temas LGTBI incluye alguna mención a partidos políticos, de tono crítico, y solo dos de ellos se refieren a Vox, formación a la que cita junto con el resto de partidos políticos (PP, PSOE, Ciudadanos y Podemos) en un mensaje relacionado con la oposición a la ley LGTBI de Castilla y León.

En el caso de $\mathrm{HO}$, el número de menciones a partidos políticos es mayor y se detecta en el $38 \%$ de sus tuits. Un porcentaje menor (8\%) se corresponde con las publicaciones en las que se menciona a Vox. Esta alusión acostumbra a darse conjuntamente con referencias a PP y Ciudadanos (en tuits en los que les reclaman que introduzcan el PIN parental en las comunidades autónomas en las que gobiernan) o con el PP (al que piden, junto a Vox, que no pacte con Ciudadanos si este no se compromete a derogar normativas LGTBI). No obstante, en seis publicaciones también se observa la presencia aislada de Vox, normalmente en tuits de tono positivo relacionados con la participación de diputados del partido de ultraderecha en actividades organizadas por $\mathrm{HO}$, la concesión de premios de esta entidad a representantes de la formación política, como Francisco Serrano, o la recomendación del voto a este partido tras examinar sus propuestas.

\section{Discusión y conclusiones}

El mensaje neocón en torno a las personas LGTBI muestra un notorio carácter defensivo. La estrategia utilizada por los tres actores estudiados, dos de perfil social (HazteOir.org y Foro Español de la Familia) y un tercero político (Vox), coincide en responder, sobre todo en los dos primeros casos, a una lógica victimista que facilita alterar los términos de la relación de poder estructural y sistémica (refrendada, además, por los datos oficiales de la discriminación y violencia que sufre la población LGTBI) entre sujetos opresores y sujetos oprimidos, entre los enunciadores del discurso y aquellos a los que interpela, de manera más o menos directa. La inversión de la vulnerabilidad que se logra por medio de un relato que representa los avances hacia la igualdad de las personas LGTBI en términos de adoctrinamiento infantil, recorte de derechos y discriminación de mayorías supone redefinir quién acosa a quién.

El análisis de la transitividad del discurso de estos actores permite entender qué posición ocupan y qué papel juegan los participantes que aparecen en sus publicaciones. Ellos, como enunciadores desde la posición de víctimas, lo- 
gran articular un mensaje aparentemente legítimo -de respuesta a una agresión- y esquivar, al mismo tiempo, un discurso abiertamente LGTBIfóbico, hasta el punto de que llegan a expresar que "la homofobia no es ningún valor. Y no somos homófobos" (FEF, I6/02/2019). Las personas LGTBI, y cualquier aliado en la lucha por su igualdad, suponen una clara amenaza a la mayoría social. De este modo, el mensaje parece tener como objetivo evitar la imagen de quedar ligado a una minoría de radicales y, por tanto, ser leído como una estrategia de confrontación que, según algunos autores (Cornejo \& Pichardo, 20I8), habría dificultado que estas voces antigénero lograran movilizaciones eficaces en los últimos años. Sin embargo, la estrategia de autovictimización no es nueva en los actores sociales conservadores, sino que constituye un recurso clave de su repertorio simbólico que, reactivado cada cierto tiempo, logra evocar la resonancia cultural de la persecución religiosa, tan hábilmente explotada en la retórica que acompañó algunos episodios en los que la transformación social del país arriesgaba la hegemonía cultural de ciertas instituciones en ámbitos como el educativo (Carratalá, 2020).

La construcción del discurso desde un artificial espacio de vulnerabilidad permite atenuar el carácter discriminatorio y contrario a los derechos de la población LGTBI del mensaje de estos actores. Su oposición a la protección, visibilidad e igualdad de las personas LGTBI, en un contexto de impulso de legislación con ese objetivo, se concreta en una comunicación que sortea la retórica con la que habitualmente se identifica el discurso de odio, explícitamente vejatorio, difamatorio y degradante. No obstante, la carga negativa que se desprende de sus significados implícitos y presuposiciones, como la insistencia en hablar de un lobby LGTBI, debiera ser objeto de atención dado que favorece incrementar el mensaje discriminatorio en un foro, el de las redes sociales, en el que, según registros oficiales, el discurso de odio encuentra una de sus vías de expresión más prósperas.

La llegada de Vox a las instituciones ha permitido la entrada de este discurso en sede parlamentaria. A través de Twitter, sin embargo, los mensajes de la formación política aluden con menor frecuencia a cuestiones LGTBI en comparación con los actores sociales estudiados. Para la formación de ultraderecha, la crítica a la llamada ideología de género se concreta, sobre todo, en su oposición a las leyes sobre violencia machista y al feminismo. En uno de sus tuits (20/0I/2020), de hecho, el concepto ideología de género queda representado con un emoticono del símbolo de la mujer, mientras que, por ejemplo, el FEF opta por ilustrarlo con la bandera del arcoíris (II/I2/20I9), símbolo de la lucha por los derechos LGTBI. En todo caso, la alusión al actor 
político, ya con representación institucional en España, en el discurso de los actores sociales, sobre todo por parte de HO, apunta al reconocimiento de un poder legitimado democráticamente en el que anclar un discurso cuestionador de derechos fundamentales.

\section{Bibliografía}

Alabao, Nuria. 202I. Las guerras de género: La extrema derecha contra el feminismo. En Ramos, Miquel (coord.) De los neocon a los neonazis: La derecha radical en el estado español. Madrid: Fundación Rosa Luxemburgo, 397-423.

Bardin, Laurence. 1986. El análisis de contenido. Torrejón de Ardoz: Akal.

Carratalá, Adolfo. 2020. Resonancia cultural y consonancia informativa como elementos favorecedores de la simbiosis discursiva entre activismo y periodismo en protestas educativas. Index.comunicación IO(I): 43-74.

Carratalá, Adolfo. 20I3. La representación de la protesta contra Educación para la Ciudadanía en ABC y La Razón. Ámbitos: Revista Internacional de Comunicación 23: I-29.

Carratalá, Adolfo \& Herrero-Jiménez, Beatriz. 20I9. La regulación contra el discurso de odio hacia el colectivo LGTBI en los medios: Análisis comparado de diez leyes autonómicas. RAE-IC: Revista de la Asociación Española de Investigación de la Comunicación 6(12): 58-80.

Castillo Jara, Soledad; Marchena Montalvo, Valerie \& Quiliche Arévalo, Sandra. 2019. El discurso antifeminista como recurso electoral en Brasil y España. Politai: Revista de Ciencia Política 19: 35-59.

Cohen, Stanley. 2004. Folk Devils \& Moral Panics. London: Routledge.

Comas d'Argemir, Dolors. 20I9. L'antifeminisme de l'extrema dreta i la ideologia de gènere. Nous Horitzons 221: 48-53.

Cornejo, Mónica \& Pichardo, J. Ignacio. 20I8. Actores y estrategias en la movilización anti-género en España: El desplazamiento de una política de iglesia al activismo laico. Revista Psicologia Política I8(43): 524-542.

Cornejo, Mónica \& Pichardo, J. Ignacio. 20I7. From the pulpit to the streets: Ultra-conservative religious positions against gender in Spain. En Kuhar, Roman \& Paternotte, David (eds.) Anti-gender campaigns in Europe: Mobilizations against Equality. London: Rowman \& Littlefield, 233-252.

Critcher, Chas. 2003. Moral Panics and the Media. Maidenhead: Open University Press.

Cuesta, Román. 2021. Fundamentalismo religioso. En Ramos, Miquel (coord.) De los neocon a los neonazis: La derecha radical en el estado español. Madrid: Fundación Rosa Luxemburgo, 272-335.

Datta, Neil. 20I8. Restaurar el orden natural. Bruselas: Foro Parlamentario Europeo sobre Población y Desarrollo.

Díaz-Salazar, Rafael. 2007. Democracia laica y religión pública. Madrid: Taurus. 
Díaz-Salazar, Rafael. 2005 (28 de febrero). La estrategia de los “neocon” en la Iglesia. La Vanguardia, 24.

Fairclough, Norman. I995. Media Discourse. London: Edward Arnold.

Ferreira, Carles. 20I9. Vox como representante de la derecha radical en España: Un estudio sobre su ideología. Revista Española de Ciencia Política 51: 73-98.

Fillieule, Olivier \& Broqua, Christophe. 2020. Sexual and reproductive rights movements and counter movements from an interactionist perspective. Social Movement Studies I9(I): I-20.

Fowler, Roger. I991. Language in the News: Discourse and Ideology in the Press. London: Routledge.

Graff, Agnieszka; Kapur, Ratna \& Walters, Suzanna Danuta. 20I9. Introduction: Gender and the rise of the global right. Signs: Journal of Women in Culture and Society 44(3): 54I-560.

Juste, Adrián. 202I. Medios de comunicación y extrema derecha. En Ramos, Miquel (coord.) De los neocon a los neonazis: La derecha radical en el estado español. Madrid: Fundación Rosa Luxemburgo, 337-358.

Kimmel, Michael. 20I9. Hombres (blancos) cabreados. València: Barlin Libros.

Lakoff, George. 2007. No pienses en un elefante: Lenguaje y debate político. Madrid: Editorial Complutense.

Luque Ortiz, Sergio \& Cano Alarcón, Mónica. 202I. La violencia de género en Twitter según Vox en las elecciones autonómicas de Andalucía. Ámbitos: Revista Internacional de Comunicación 51: II6-I33.

Mayer, Stefanie, Šori, Iztok \& Sauer, Birgit. 20I6. Gendering 'the People'. Heteronormativity and 'Ethnomasochism' in Populist Imaginary. En Ranieri, Maria (ed.) Populism, Media, and Education: Challenging Discrimination in Contemporary Digital Societies. New York: Routledge, 84-I04.

Michavila, Narciso. 20I9. ¿De dónde salen sus 400.000 votos? Perfil sociológico del votante de Vox. En Müller, John (coord.) La sorpresa de Vox: Las respuestas a las 10 grandes preguntas que todos nos hacemos sobre Vox. Barcelona: Planeta, 28-4I.

Ministerio del Interior. 202I. Informe sobre la evolución de los delitos de odio en España 2020. http://www.interior.gob.es/documents/642012/1362247I/Informe+sobre+la+evoluci\%C3\%B3n+de+delitos+de+odio+en+Espa $\% \mathrm{C}_{3} \% \mathrm{~B}$ га $+\mathrm{a} \% \mathrm{C}_{3} \%$ Вio+2020.pdf/bc4738d2-ebe6-434f-9516-5d511a894cb9

Paternotte, David. 20I5. Blessing the crowds: Catholic mobilizations against gender in Europe. En Hark, Sabine \& Irene-Villa, Paula (eds.) Anti-Genderismus. Sexualität und Geschlecht als Schauplätze aktueller politischer Auseinandersetzungen. Bielfeld: Transcript Verlag, I29-I47.

Paternotte, David \& Kuhar, Roman. 20I8. Disentangling and locating the "global right": Anti-gender campaigns in Europe. Politics and Governance 6(3): 6-I9.

Ramos, Miquel. 202I. La irrupción de Vox. En Ramos, Miquel (coord.). De los neocon a los neonazis: La derecha radical en el estado español. Madrid: Fundación Rosa Luxemburgo, 33-I24.

Richardson, John E. 2007. Analysing Newspapers: An Approach from Critical Discourse Analysis. New York: Palgrave Macmillan. 
Rivas Venegas, Miguel. 202I. Contra las mujeres: El discurso misógino de Vox. "Palabras gruñido” del nacional-populismo español. Investigaciones Feministas I2(I): 67-77.

Ruzza, Carlo. 202I. The institutionalisation of populist political discourse and conservative uncivil society in the European Union: From the margins to the mainstream? Nordicom Review 42(SI): II9-I33.

Simancas González, Esther \& Marín Dueñas, Pedro Pablo. 20I9. Influencia de Twitter en la prensa digital en el caso de la campaña de Hazte Oír: "Los niños tienen pene y las niñas tienen vulva”. Revista Inclusiones 6: 152-I67.

Tamayo, Juan José. 202I (23 de marzo). El cristoneofascismo, nueva religión. El País, I2.

Van Dijk, Teun A. 20ı6. Análisis Crítico del Discurso. Revista Austral de Ciencias Sociales 30: 203-222.

Villar-Aguilés, Alícia \& Pecourt Gracia, Juan. 2021. Antifeminismo y troleo de género en Twitter. Estudio de la subcultura trol a través de \#STOPfeminazis. Teknokultura I8(I): 33-44.

Wilcox, Clyde \& Larson, C. 2006. Onward Christian Soldiers?: The Religious Right in American Politics. Boulder: Westview Press. 